\title{
Insights Into the Results of Sotagliflozin Cardiovascular Outcome Trials: Is Dual Inhibition the Cherry on the Cake of Cardiorenal Protection?
}

\author{
Theocharis Koufakis ${ }^{1} \cdot$ Omar G. Mustafa ${ }^{2} \cdot$ Vasilios Tsimihodimos $^{3} \cdot$ Ramzi A. Ajjan ${ }^{4} \cdot$ Kalliopi Kotsa $^{1}$
}

Accepted: 20 June 2021 / Published online: 7 July 2021

(c) The Author(s), under exclusive licence to Springer Nature Switzerland AG 2021

\begin{abstract}
Sotagliflozin is a dual sodium-glucose co-transporter (SGLT) 2 inhibitor, manifesting a 20-fold higher inhibitory activity for SGLT2 than for SGLT1. Differences in SGLT2 over SGLT1 selectivity of the available agents have been proposed to relate to variability in efficacy and safety characteristics. In contrast to other SGLT2 inhibitors, the cardiorenal effects of sotagliflozin in type 2 diabetes had not been explored until recently, when the results of SOLOIST-WHF (focusing on heart failure [HF] outcomes) and SCORED (focusing on renal outcomes) were published. In SOLOIST-WHF, sotagliflozin reduced the risk of the primary composite outcome of cardiovascular (CV) death and hospitalizations and urgent visits for HF. The findings showed that the risk reduction was consistent in people with reduced but also in those with preserved ejection fraction (EF). In SCORED, sotagliflozin significantly reduced the primary end point of CV deaths, hospitalizations for HF, and urgent visits for HF. A reduction in glycated hemoglobin was evident even in participants with estimated glomerular filtration rate values below $30 \mathrm{~mL} / \mathrm{min} / 1.73 \mathrm{~m}^{2}$. SCORED is also the first trial to illustrate the benefits of the class across the full range of albuminuria. Moreover, the endpoint of stroke was significantly reduced by $34 \%$ in the sotagliflozin compared with the placebo group. The findings of the two studies provide novel insights into the clinical utility of SGLT2 inhibitors, particularly with respect to the early initiation in stable HF, the benefits in HF with preserved EF, the glucose-lowering efficacy in people with severe renal impairment and their potential to improve atherosclerotic vascular disease, including stroke, outcomes.
\end{abstract}

Kalliopi Kotsa

kalmanthou@yahoo.gr

1 Division of Endocrinology and Metabolism and Diabetes

Center, First Department of Internal Medicine,

Medical School, Aristotle University of Thessaloniki, AHEPA University Hospital, 1 St. Kiriakidi Street, 54636 Thessaloniki, Greece

\section{Introduction}

2 Department of Diabetes, King's College Hospital, London, UK

3 Department of Internal Medicine, School of Medicine, University of Ioannina, Ioannina, Greece

4 Leeds Institute of Cardiovascular and Metabolic Medicine, University of Leeds, Leeds, UK

\section{Key Points}

SOLOIST-WHF and SCORED explored the effects of sotagliflozin on cardiorenal outcomes.

The results reconfirm the class effects of SGLT2i but also differentiate sotagliflozin from other agents.

Dual SGLT inhibition might contribute to the unique safety and efficacy profile of the drug.

Sodium-glucose co-transporter (SGLT) 2 inhibitors (SGLT2i) lower blood glucose by inhibiting SGLT2 expressed in the proximal renal tubule, thus modulating the excessive renal glucose reabsorption typically observed 
in people with type 2 diabetes (T2D) [1]. Therefore, their mechanism of action differs from traditional agents that lower blood glucose, which either focus on alleviating insulin resistance or improving beta-cell function. The use of SGLT2i has been linked to metabolic benefits, including improvements in glycated hemoglobin (HbAlc), reduction in body weight and blood pressure, with minimal risk of hypoglycemia [2]. However, the revolutionary impact of the class on T2D management has not been driven by its metabolic effects. It is the potential of SGLT2i (along with that of glucagon-like peptide 1 [GLP-1] receptor agonists) for improving cardiac and renal outcomes, that gradually moved diabetes care from a strictly glucose-centric approach to the notion that the burden of disease complications should be simultaneously addressed with hyperglycemia.

Specifically, a number of large-scale cardiovascular outcome trials (CVOTs) have demonstrated a reduced risk of major cardiovascular (CV) events, hospitalization for heart failure (HF), CV death, and renal outcomes in people treated with SGLT2i [3]. Although these properties are particularly evident at the secondary prevention level, a recently published post hoc analysis of DECLARE-TIMI (conducted with dapagliflozin) demonstrated similar improvements in $\mathrm{HF}$ and kidney outcomes between the primary prevention cohort and the group with established CV disease [4]. While the full spectrum of the mechanisms implicated in the cardiorenal benefits of SGLT2i are still under investigation, these are likely to be multifactorial, which explains the large number of implicated pathways [5].

\section{Dual Inhibition}

Sotagliflozin is a dual SGLT2i, manifesting a 20 -fold higher inhibitory activity for SGLT2 than for SGLT1. The former is predominantly expressed in the apical membrane of renal proximal convoluted tubules (S1 and S2 segments), whereas the latter is located in the straight renal proximal tubule (S3 segment), but also in other human tissues, including the brush-border membrane of the small intestine, heart, and brain [6]. Differences in SGLT2 over SGLT1 selectivity of the available agents have been proposed to relate to variability in efficacy and safety characteristics. For instance, blocking SGLT1 might provide additional glucose-lowering efficacy through the inhibition of both intestinal and renal absorption of glucose and augmented release of GLP-1, and protect cardiac tissue by reducing glycogen accumulation [7]. On the other hand, it might increase the probability of gastrointestinal side effects [8]. In contrast to other SGLT2is, the cardiorenal effects of sotagliflozin in T2D had not been explored. It is only recently that the results of two randomized controlled trials (RCTs), SOLOIST-WHF [9] (focusing on HF outcomes) and SCORED [10] (focusing on renal outcomes) were published.

\section{SOLOIST-WHF}

In the SOLOIST-WHF trial, 1222 people with T2D who were recently hospitalized for worsening HF were randomized to receive sotagliflozin $(n=608)$ or placebo $(n=$ 614 ) and were followed for a median of 9 months. Median estimated glomerular filtration rate (eGFR) values in the sotagliflozin and placebo group were 49.2 and $50.5 \mathrm{~mL} /$ $\min / 1.73 \mathrm{~m}^{2}$, respectively, whereas patients with eGFR $<30$ $\mathrm{mL} / \mathrm{min} / 1.73 \mathrm{~m}^{2}$ were excluded. Unlike previous SGLT2i trials, sotagliflozin was initiated soon after an episode of decompensated HF, namely, either before $(48.8 \%$ of the study population) or within 3 days of hospital discharge. It was demonstrated that sotagliflozin reduced the risk of the primary composite outcome of $\mathrm{CV}$ death and hospitalizations and urgent visits for HF (first and subsequent events) by a relative $33 \%$ compared with placebo (hazard ratio [HR], 0.67 ; $95 \%$ confidence interval [CI] $0.52-0.85, p<0.001$ ).

A closer look at the findings of the trial reveals some interesting insights: first, the risk reduction came surprisingly early, achieving significance by 28 days of follow-up [11]. Second, efficacy and safety seemed to be similar in those who received sotagliflozin shortly before discharge, following stabilization, or in the following 3 days. Taken together, these data support an "earlier, better" approach in initiating therapy with SGLT2i in clinically stable HF, even prior to hospital discharge. Such a strategy could enable proper dose adjustment of diuretics or antihypertensive medications and enhance treatment adherence [5]. It could also help to tackle clinical inertia, considering available data showing that among people with HF and T2D managed in primary care settings, SGLT2is are only prescribed in a small proportion of patients, contrary to available evidence [12].

Third, SGLT2i trials focused on HF-related outcomes have so far included people with reduced $(\leq 40 \%)$ ejection fraction $(E F)$, demonstrating a benefit of treatment with dapagliflozin [13] and empagliflozin [14]. However, there is limited evidence on the effects of SGLT2i in individuals with $\mathrm{HF}$ and preserved $\mathrm{EF}$ (HFpEF), although ongoing trials (DELIVER for dapagliflozin and EMPEROR-Preserved for empagliflozin) are expected to shed light on this issue. The findings of SOLOIST-WHF showed that the risk reduction was consistent in people with reduced but also in those with preserved EF (approximately $21 \%$ of the study participants), providing important data demonstrating that SGLT2is have the potential to improve HF outcomes across the range of EFs. More specifically, the HR for the primary outcome was similarly reduced in all patient subgroups: 0.69 for 
those with EF $<40 \%, 0.74$ for those with EF 40-49\% and 0.66 for those with $\mathrm{EF} \geq 50 \%$. In a rat model of metabolic HFpEF, sotagliflozin has been shown to ameliorate left-atrial remodeling by preventing mitochondrial swelling and reducing production of reactive oxygen species [15]. However, from a strictly mechanistic perspective, whether dual SGLT inhibition could confer additional benefits on patients with HFpEF compared with selective SGLT2i, remains unclear at the moment.

\section{SCORED}

In the SCORED trial, 10,584 people with T2D and chronic kidney disease (CKD) (eGFR 25-60 mL/min/1.73 $\mathrm{m}^{2}$ ) were randomly assigned to receive sotagliflozin $(n=5292)$ or placebo $(n=5292)$ and followed for a median of 16 months. Median eGFR values in the sotagliflozin and placebo group were 44.4 and $44.7 \mathrm{~mL} / \mathrm{min} / 1.73 \mathrm{~m}^{2}$, respectively. Sotagliflozin significantly reduced the primary end point (PEP) of CV deaths, hospitalizations for $\mathrm{HF}$, and urgent visits for HF (HR 0.74, 95\% CI 0.63-0.88, $p<0.001$ ). Although the PEP was changed during the trial, the original endpoints were significantly in favor of sotagliflozin: first occurrence of $\mathrm{CV}$ death, nonfatal myocardial infarction, or nonfatal stroke (HR 0.84, 95\% CI 0.72-0.99) and the first occurrence of $\mathrm{CV}$ death or hospitalization for $\mathrm{HF}$ (HR 0.77, 95\% CI
0.66-0.91). These data are in line with the findings of the previous SGLT2i CVOTs, suggesting that the basic cardiorenal benefits of these drugs are perhaps a class effect and not related either to SGLT1 or SGLT2 inhibition alone [16].

Figure 1 summarizes the design and the primary outcome findings of SOLOIST-WHF and SCORED.

\section{Insights into the Results of SOLOIST-WHF and SCORED in the Context of Previous SGLT2i Trials}

DAPA-HF [13] and EMPEROR-Reduced [14] recruited ambulatory patients with $\mathrm{HF}$ and reduced EF with or without T2D. Differently, in SOLOIST-WHF, the study population comprised recently hospitalized individuals who all had T2D and covered the entire spectrum of EFs. Participants in the latter trial were at higher risk for $\mathrm{CV}$ events, as indicated by the placebo event rate for first $\mathrm{CV}$ death or hospitalization for HF, which was $48 \%$ in SOLOIST-WHF compared to $25.5 \%$ and $28.5 \%$ in DAPA-HF and EMPEROR-Reduced, respectively. These data emphasize the potential of sotagliflozin to reduce $\mathrm{CV}$ outcomes in a very high-risk population with T2D and HF, which has not been adequately represented in other SGLT2i trials, so far.

Interestingly, in the SCORED trial, 419 participants (7.9\%) in the intervention and 394 participants $(7.4 \%)$ in the

\section{SOLOIST-WHF}

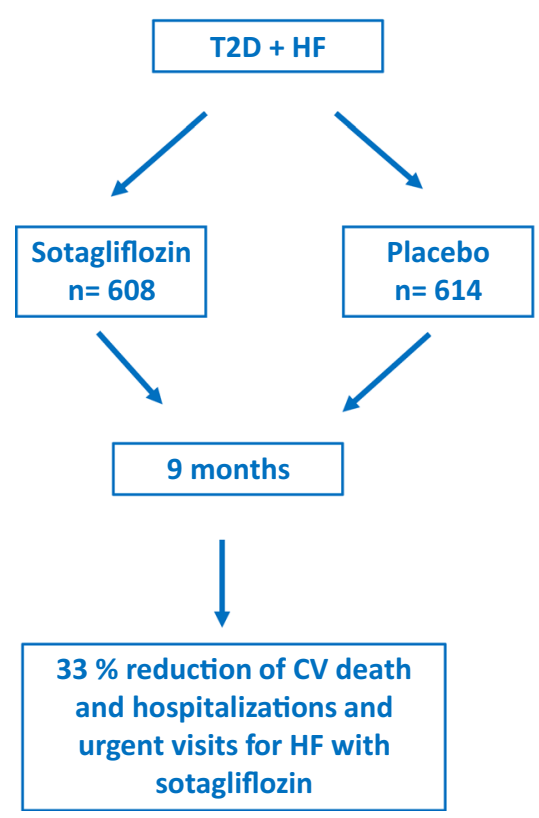

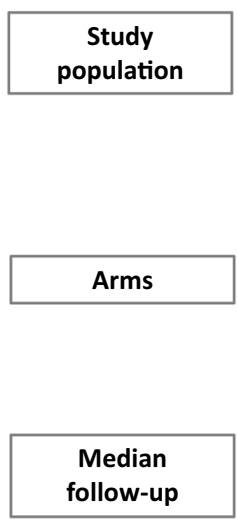

Primary outcome

\section{SCORED}

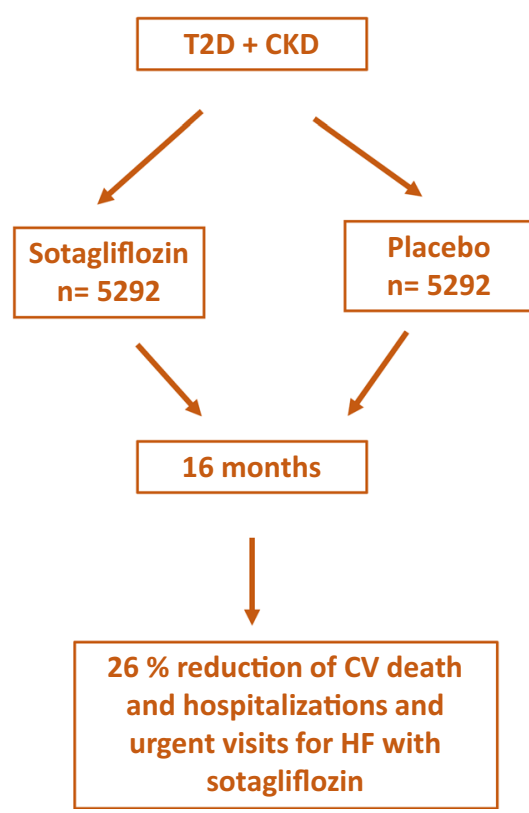

Fig. 1 Design and primary outcome findings of SOLOIST-WHF and SCORED. $C K D$ chronic kidney disease, $C V$ cardiovascular, $H F$ heart failure, $T 2 D$ type 2 diabetes 
placebo group had eGFR values lower than $30 \mathrm{~mL} / \mathrm{min} / 1.73$ $\mathrm{m}^{2}$. Individuals with eGFR lower than $30 \mathrm{~mL} / \mathrm{min} / 1.73$ $\mathrm{m}^{2}$ have been generally underrepresented in CVOTs, given that they had been totally excluded from CREDENCE [17] and while included in DAPA-CKD [18], they represented only $14 \%$ of the entire study population. Thus, they comprise a population with few data on SGLT2i use.

There are some aspects of the results of the SCORED trial that deserve a more detailed look in the context of the dual SGLT inhibition. In the EMPA-REG OUTCOME trial [19], stroke incidence with empagliflozin treatment was numerically, albeit not statistically, increased, despite the reduction in overall $\mathrm{CV}$ mortality. A post hoc analysis of the trial concluded that the numeric difference between the two arms was primarily attributable to a small number of patients in the empagliflozin group with a first event taking longer than 3 months after cessation of the study drug [20]. However, the results of EMPA-REG OUTCOME generated a discussion about the existence of a "stroke paradox" with SGLT2i and its potential explanation, attributed either to a "play of chance" or to changes in human physiology induced by these agents, including an increase in hematocrit levels, that could predispose to the development of stroke [21]. Other largescale studies with different agents in the class showed either a numerical decrease in stroke (CANVAS, using canagliflozin) [22] or a completely neutral effect (DECLARE-TIMI, using dapagliflozin) [23], although the latter trial was conducted in a lower-risk population. In SCORED however, the endpoint of stroke was significantly reduced by $34 \%$ in the sotagliflozin compared with the placebo group and this is the first SGLT2i CVOT to establish such a benefit, after considering the different population studied and that this was demonstrated in a post hoc analysis. Given animal data linking SGLT1 downregulation to delaying of cerebral damage during traumatic brain injury [24], it could be hypothesized that the beneficial effects of sotagliflozin on stroke outcomes might be related to SGLT1 inhibition. However, this hypothesis should be tested in further studies.

In contrast to SCORED, both CREDENCE [17] and DAPA-CKD [18] have required the presence of macroalbuminuria for inclusion over and above reduced eGFR. Therefore, SCORED is the first trial to illustrate the benefits of the class across the full range of albuminuria. This observation is of clinical importance, as it substantially expands the population in which SGLT2i could improve kidney outcomes. Furthermore, the traditional knowledge about the glucoselowering effects of SGLT2i indicates that their hypoglycemic potency is greatly dependent on kidney function and progressively declines as eGFR falls. In SCORED however, a reduction in $\mathrm{HbAlc}$ was evident even in participants with eGFR values below $30 \mathrm{~mL} / \mathrm{min} / 1.73 \mathrm{~m}^{2}$, probably secondary to reduced intestinal absorption of glucose as a result of SGLT1 inhibition. Canagliflozin, the other dual SGLT inhibitor, is more selective to SGLT2 than SGLT1 compared with sotagliflozin (250-fold vs 20 -fold, respectively). As a result, its antihyperglycemic effect also decreases with advanced CKD [25]. Other glucose-lowering agents acting on the gut, such as acarbose, should be avoided in CKD stages 4 and 5, due to limited safety data [26]. Moreover, acarbose has modest efficacy on glycemic control and lacks cardiorenal benefits. Taken together, these data suggest that sotagliflozin could offer parallel cardiorenal protection and glucose-lowering action in people with advanced CKD, a population where the options of antidiabetic treatment are limited. Bridging the results of the two sotagliflozin trials could lead to the assertion that dual SGLT inhibitors should be considered early in the context of worsening HF and evidence of progressive renal disease.

\section{Pooled Data from SCORED and SOLOIST-WHF}

In a prespecified pooled analysis [27] of patient-level data from SCORED and SOLOIST-WHF, which included 11,784 participants, sotagliflozin significantly reduced the composite PEP of total CV deaths, hospitalizations for HF, and urgent visits for HF. Specifically, at 24 months' followup, total PEP events for sotagliflozin versus placebo were 15.5 versus 21.1 per 100 patient-years (HR $0.72,95 \%$ CI $0.63-0.82 ; p=0.000002$ ). This was evident even in patients with $\mathrm{HFpEF}$ ( $\mathrm{EF} \geq 50 \%, n=739$ ), in whom the rates of PEP events per 100 patient-years for sotagliflozin versus placebo were 37.5 versus 59.0 (HR 0.63 , 95\% CI $0.45-0.89 ; p=$ $0.009)$. It is worth noting that the benefits of treatment were consistent independent of sex (particularly for women with normal EF) or history of HF (HR 0.73; 95\% CI 0.54-0.99; $p=0.04$ for those with no HF history).

\section{Safety Outcomes: Does Dual Inhibition Play a Role?}

With respect to safety outcomes and in accordance with the general adverse event profile of the class, participants in the sotagliflozin group of the two trials experienced a greater number of genital mycotic infections compared to those enrolled in the placebo arm ( 2.4 vs $0.9 \% ; p<0.001$ in SCORED and 0.8 vs $0.2 \% ; p=0.12$ in SOLOIST-WHF). Despite the low eGFR of the trial population, acute kidney injury rates in SCORED were similar comparing the two arms ( 2.2 vs $2.1 \% ; p=0.55$ ), further confirming safety of SGLT2i use in renal impairment. Although relevant data are currently unavailable, it would be useful to know whether the renal safety profile of sotagliflozin 
is consistent across various eGFR categories and especially among patients in the lowest range $\left(<30 \mathrm{~mL} / \mathrm{min} / 1.73 \mathrm{~m}^{2}\right)$.

Diarrhea was also more common among individuals treated with sotagliflozin than in those receiving placebo $(8.5 \mathrm{vs}$ $6.0 \%, p<0.0001$ in SCORED and 6.9 vs $4.1 \%, p=0.032$ in SOLOIST-WHF), related to the drug's mechanism of action and its effects on the brush border of the small intestine. Only in SCORED, diabetic ketoacidosis was more common with sotagliflozin than with placebo $(0.6 \%$ vs $0.3 \%, p=0.02)$. There was no increased risk of lower extremity amputations associated with sotagliflozin treatment in either trial, suggesting that the relationship observed in CANVAS was a random observation, rather than a consequence of dual inhibition [28].

Incidence of severe hypoglycemia in the sotagliflozin group was similar between SCORED and SOLOIST-WHF (1.0\% vs $1.5 \%$ ), although the different populations studied in the two trials should be considered. On the other hand, severe hypoglycemia rates in SOLOIST-WHF were greater in the intervention than in the placebo group ( 1.5 vs $0.3 \%, p=0.037)$. Although still inconclusive and largely speculative, preliminary data indicate that SGLT1 inhibition may interfere with glucose uptake by neurons and myocytes under hypoglycemic or hypoxemic conditions, resulting in increased hypoglycemic

Table 1 Key characteristics and findings of SGLT2 inhibitor cardiovascular outcome trials

\begin{tabular}{|c|c|c|c|c|c|c|}
\hline Drug & Study acronym & $N$ & Median follow-up & $\begin{array}{l}\text { Study population } \\
\text { (mean values) }\end{array}$ & Primary outcome & $\begin{array}{l}\text { HR }(95 \% \mathrm{CI}) \\
\text { SGLT2 inhibitor vs } \\
\text { placebo }\end{array}$ \\
\hline \multirow[t]{2}{*}{ Canagliflozin } & CANVAS & 10,142 & $188.2 \mathrm{w}$ & $\begin{array}{l}\text { HbA1c: } 8.2 \% \text {; BMI: } \\
\text { 32; eGFR: 76.5; } \\
\text { CVD:65.6\%; HF: } \\
14.4 \%\end{array}$ & 3-P MACE & $0.86 ; 0.75-0.97$ \\
\hline & CREDENCE & 4401 & $2.6 \mathrm{y}$ & $\begin{array}{l}\text { HbA1c: } 8.3 \% \text {; BMI: } \\
\text { 31.3; eGFR: 56.2; } \\
\text { CVD:50.4\%; HF: } \\
14.8 \%\end{array}$ & $\begin{array}{l}\text { ESRD, doubling serum } \\
\text { creatinine, renal or } \\
\text { CV death (composite) }\end{array}$ & $0.70 ; 0.59-0.82$ \\
\hline \multirow[t]{3}{*}{ Dapagliflozin } & DECLARE-TIMI & 17,160 & $4.2 \mathrm{y}$ & $\begin{array}{l}\text { HbA1c: } 8.3 \% \text {; BMI: } \\
\text { 32.1; eGFR: 85.2; HF: } \\
\text { 11.6\% (HFrEF: 7.7\%) }\end{array}$ & $\begin{array}{l}\text { 3-P MACE/CV death or } \\
\text { HHF (composite) }\end{array}$ & $\begin{array}{l}0.93 ; 0.84-1.03 \text { / } 0.83 \\
\quad 0.73-0.95\end{array}$ \\
\hline & DAPA-HF & 4744 & $18.2 \mathrm{~m}$ & $\begin{array}{l}\text { T2D: } 41.8 \% \text {; BMI: } \\
\text { 28.2; eGFR 65.8; } \\
\text { HFrEF: } 100 \%\end{array}$ & $\begin{array}{c}\text { Worsening HF or CV } \\
\text { death (composite) }\end{array}$ & $0.74 ; 0.65-0.85$ \\
\hline & DAPA-CKD & 4304 & $2.4 \mathrm{y}$ & $\begin{array}{l}\text { T2D: } 67.5 \% \text {; BMI: } \\
\text { 29.5; eGFR: 43.9; HF: } \\
\text { 10.9\% }\end{array}$ & $\begin{array}{l}\text { Sustained } \geq 50 \% \\
\text { decline in GFR, } \\
\text { ESRD, renal death or } \\
\text { CV death (composite) }\end{array}$ & $0.61 ; 0.51-0.72$ \\
\hline \multirow[t]{2}{*}{ Empagliflozin } & EMPA-REG & 7020 & $3.1 \mathrm{y}$ & $\begin{array}{l}\text { HbA1c: } 8.1 \% \text {; BMI: } \\
\text { 30.6; HF: 10.1\%; } \\
\text { eGFR: 74; CVD: } \\
\text { 100\% }\end{array}$ & 3-P MACE & $0.86 ; 0.74-0.99$ \\
\hline & EMPEROR-Reduced & 3730 & $18 \mathrm{~m}$ & $\begin{array}{l}\text { T2D: 49.8\%; BMI: } \\
\text { 27.9; eGFR 62; } \\
\text { HFrEF: } 100 \%\end{array}$ & $\begin{array}{l}\text { CV death or HHF } \\
\text { (composite) }\end{array}$ & $0.75 ; 0.65-0.86$ \\
\hline Ertugliflozin & VERTIS-CV & 8246 & $3.5 \mathrm{y}$ & $\begin{array}{l}\text { HbA1c: } 8.2 \% \text {; BMI: } \\
\text { 32; eGFR: 76; CAD: } \\
\text { 76.3\%; HF: } 23.1 \%\end{array}$ & 3-P MACE & $0.97 ; 0.85-1.11$ \\
\hline \multirow[t]{2}{*}{ Sotagliflozin } & SOLOIST-WHF & 1222 & $9 \mathrm{~m}$ & $\begin{array}{l}\text { HbA1c: } 7.1 \% \text {; BMI: } \\
\text { 30.4; eGFR: 49.2; } \\
\text { HFrEF: 79\%; drug } \\
\text { initiation before hos- } \\
\text { pital discharge: } 48.8 \%\end{array}$ & $\begin{array}{l}\text { CV death, HHF or } \\
\text { urgent visits for HF } \\
\text { (composite) }\end{array}$ & $0.67 ; 0.52-0.85$ \\
\hline & SCORED & 10,584 & $16 \mathrm{~m}$ & $\begin{array}{l}\text { HbA1c: } 8.3 \% \text {; BMI: } \\
\text { 31.9; eGFR: 44.4; HF: } \\
\text { 31\%; HFrEF: } 19 \%\end{array}$ & $\begin{array}{l}\text { CV death, HHF or } \\
\text { urgent visits for HF } \\
\text { (composite) }\end{array}$ & $0.74 ; 0.63-0.88$ \\
\hline
\end{tabular}

3-P MACE 3-point major adverse cardiovascular events, $B M I$ body mass index (units: $\mathrm{kg} / \mathrm{m}^{2}$ ), $C A D$ coronary artery disease, $C I$ confidence interval, $C V$ cardiovascular, $C V D \mathrm{CV}$ disease, $e G F R$ estimated glomerular filtration rate (units: $\mathrm{mL} / \mathrm{min} / 1.73 \mathrm{~m}{ }^{2}$ ), ESRD end-stage renal disease, $H b A l c$ glycated hemoglobin, $H F$ heart failure, $H F r E F$ HF with reduced ejection fraction, $H H F$ hospitalization for heart failure, $H R$ hazard ratio, $m$ months, $N$ Number of participants, $S G L T 2$ sodium-glucose co-transporter 2, T2D type 2 diabetes, $w$ weeks, $y$ years 
risk under treatment with dual inhibitors [29]. High diarrhea rates in the intervention group (8.5\% in SCORED and 6.1\% in SOLOIST-WHF) might represent an interaction. Despite similar rates of hypoglycemia in canagliflozin and placebo arms in the CAVNAS trial, it could be argued that sotagliflozin manifests significantly smaller selectivity for SGLT2 over SGLT1 than canagliflozin. Thus, some of the effects of SGLT1 inhibition might be more intense with the use of the former compared to that of the latter.

In a similar way, diarrhea and exaggerated osmotic diuresis and natriuresis related to dual inhibition might be responsible for the greater volume depletion rates observed in sotagliflozin-treated individuals than the placebo group in SCORED (5.3 vs 4.0\%, respectively, $p=0.003$ ). Such an association was also evident in CANVAS ( $26 \%$ vs $18.5 \%$, $p=0.009$ ), although not evident in EMPA-REG, using empagliflozin, which has minimal SGLT1 inhibitory activity. It should be stressed, however, that direct inter-study comparisons are not always feasible, and that the observed differences in safety outcomes might not relate to individual pharmacological characteristics of the various agents, but to different patient populations as well as the definition of outcomes across various trials [16] (Table 1).

\section{Limitations}

The results of SOLOIST-WHF and SCORED should be interpreted with caution. Both trials were prematurely interrupted due to loss of funding from their main sponsors and because of the COVID-19 pandemic, which affected their PEP. The latter was expanded to include the total number of events and urgent visits for HF. This change in study protocols might have influenced the results in favor of sotagliflozin. Moreover, the studies were not adequately powered for detecting changes in secondary outcomes and subgroup analyses. Finally, non-white populations were underrepresented in both trials.

\section{Conclusion}

In conclusion, and despite their limitations, SOLOISTWHF and SCORED trials reconfirm the substantial benefits of SGLT2i in people with T2D, renal disease, and/or HF. Moreover, they provide novel insights into the clinical utility of these drugs, particularly with respect to the early initiation (even during hospitalization) in stable HF, the benefits in HF with preserved EF, the glucose-lowering efficacy in people with severe renal impairment and their potential to improve atherosclerotic vascular disease, including stroke, outcomes. It should be stressed, however, that differences in the inclusion criteria, study design, and drug characteristics between sotagliflozin and previous SGLT2i trials limit the extrapolation of the findings of SOLOIST-WHF and SCORED to other SGLT2is. Future mechanistic studies, RCTs with longer follow-up periods, and accumulation of real-world data will help to further clarify whether dual SGLT inhibition could be the "cherry on the cake" of cardiorenal protection offered by the class.

\section{Declarations}

Funding No external funds were used in the preparation of this manuscript.

Conflicts of interest TK has received honoraria for lectures/advisory boards and research support from AstraZeneca, Boehringer Ingelheim, Novo Nordisk and Eli-Lilly; OGM has received speaker honoraria and educational grants from Sanofi, Eli Lilly, Boehringer Ingelheim and Novo Nordisk; VT has received honoraria for lectures/advisory boards and research support from Astra Zeneca, Boehringer Ingelheim, Pharmaserve Lilly, Sanofi-Aventis, ELPEN, MSD, Winmedica, Servier, Abbot, and Novo Nordisk; RAA has received research/educational support and consultancy fees from Abbott Diabetes Care, AstraZeneca, Bayer, Boehringer Ingelheim, Bristol-Myers Squibb, Eli Lilly, GlaxoSmithKline, Merck Sharp \& Dohme, Menarini, NovoNordisk, Roche and Takeda; KK has received honoraria for lectures/advisory boards and research support from Astra Zeneca, Boehringer Ingelheim, Pharmaserve Lilly, Sanofi-Aventis, ELPEN, MSD, and Novo Nordisk.

Ethics approval Not applicable.

Consent to participate Not applicable.

Consent for publication Not applicable.

Availability of data and material Not applicable.

Code availability Not applicable.

Authors' contributions TK reviewed the literature and drafted the first version of the manuscript. OGM, VT, RAA and KK reviewed the literature and critically edited the manuscript. All authors have read and approved the final version of the manuscript.

\section{References}

1. DeFronzo RA, Davidson JA, Del Prato S. The role of the kidneys in glucose homeostasis: a new path towards normalizing glycaemia. Diabetes Obes Metab. 2012;14:5-14.

2. Wilding J, Fernando K, Milne N, et al. SGLT2 inhibitors in Type 2 diabetes management: key evidence and implications for clinical practice. Diabetes Ther. 2018;9:1757-73.

3. McGuire DK, Shih WJ, Cosentino F, et al. Association of SGLT2 inhibitors with cardiovascular and kidney outcomes in patients with type 2 diabetes: a meta-analysis. JAMA Cardiol. 2021;6:148-58.

4. Cahn A, Raz I, Leiter LA, et al. Cardiovascular, renal, and metabolic outcomes of dapagliflozin versus placebo in a primary cardiovascular prevention cohort: analyses from DECLARE-TIMI 58. Diabetes Care. 2021;44:1159-67. 
5. Koufakis T, Mustafa OG, Ajjan RA, et al. The use of sodiumglucose co-transporter 2 inhibitors in the inpatient setting: is the risk worth taking? J Clin Pharm Ther. 2020;45:883-91.

6. Elfeber K, Köhler A, Lutzenburg M, et al. Localization of the $\mathrm{Na}^{+}$-D-glucose cotransporter SGLT1 in the blood-brain barrier. Histochem Cell Biol. 2004;121:201-7.

7. Tsimihodimos V, Filippas-Ntekouan S, Elisaf M. SGLT1 inhibition: pros and cons. Eur J Pharmacol. 2018;838:153-6.

8. Dominguez Rieg JA, Rieg T. What does sodium-glucose co-transporter 1 inhibition add: prospects for dual inhibition. Diabetes Obes Metab. 2019;21(Suppl 2):43-52.

9. Bhatt DL, Szarek M, Steg PG, et al. Sotagliflozin in patients with diabetes and recent worsening heart failure. N Engl J Med. 2021;384:117-28.

10. Bhatt DL, Szarek M, Pitt B, et al. Sotagliflozin in patients with diabetes and chronic kidney disease. N Engl J Med. 2021;384:129-39.

11. Bhatt DL. Sotagliflozin in diabetes patients with recent worsening heart failure-SOLOIST-WHF. In: Presented at the American Heart Association 2020 Virtual Scientific Sessions; November $13-17,2020$.

12. Schernthaner G, Shehadeh N, Ametov AS, et al. Worldwide inertia to the use of cardiorenal protective glucose-lowering drugs (SGLT2i and GLP-1 RA) in high-risk patients with type 2 diabetes. Cardiovasc Diabetol. 2020;19:185.

13. McMurray JJV, Solomon SD, Inzucchi SE, et al. Dapagliflozin in patients with heart failure and reduced ejection fraction. N Engl J Med. 2019;381:1995-2008

14. Packer M, Anker SD, Butler J, et al. Cardiovascular and renal outcomes with empagliflozin in heart failure. $\mathrm{N}$ Engl $\mathrm{J}$ Med. 2020;383:1413-24.

15. Bode D, Semmler L, Wakula P, et al. Dual SGLT-1 and SGLT-2 inhibition improves left atrial dysfunction in HFpEF. Cardiovasc Diabetol. 2021;20:7.

16. Koufakis T, Papanas N, Dimitriadis G, et al. Interpreting the results of the VERTIS-CV trial: is this the end of the "class effect" perspective? J Diabetes. 2020;12:942-5.

17. Perkovic V, Jardine MJ, Neal B, et al. Canagliflozin and renal outcomes in type 2 diabetes and nephropathy. N Engl J Med. 2019;380:2295-306.
18. Heerspink HJL, Stefánsson BV, Correa-Rotter R, et al. Dapagliflozin in patients with chronic kidney disease. N Engl J Med. 2020;383:1436-46.

19. Zinman B, Wanner C, Lachin JM, et al. Empagliflozin, cardiovascular outcomes, and mortality in type 2 diabetes. N Engl J Med. 2015;373:2117-28.

20. Zinman B, Inzucchi SE, Lachin JM, et al. Empagliflozin and cerebrovascular events in patients with type 2 diabetes mellitus at high cardiovascular risk. Stroke. 2017;48:1218-25.

21. Imprialos KP, Boutari C, Stavropoulos K, et al. Stroke paradox with SGLT-2 inhibitors: a play of chance or a viscosity-mediated reality? J Neurol Neurosurg Psychiatry. 2017;88:249-53.

22. Neal B, Perkovic V, Mahaffey KW, et al. Canagliflozin and cardiovascular and renal events in type 2 diabetes. N Engl J Med. 2017;377:644-57.

23. Wiviott SD, Raz I, Bonaca MP, et al. Dapagliflozin and cardiovascular outcomes in type 2 diabetes. N Engl J Med. 2019;380:347-57.

24. Sebastiani A, Greve F, Gölz C, et al. RS1 (Rsc1A1) deficiency limits cerebral SGLT1 expression and delays brain damage after experimental traumatic brain injury. J Neurochem. 2018;147:190-203.

25. Yamout H, Perkovic V, Davies M, et al. Efficacy and safety of canagliflozin in patients with type 2 diabetes and stage 3 nephropathy. Am J Nephrol. 2014;40:64-74.

26. Williams ME, Garg R. Glycemic management in ESRD and earlier stages of CKD. Am J Kidney Dis. 2014;63:S22-38.

27. Bhatt DL, Szarek M, Steg PG, et al. Benefits of sodium glucose co-transporter-1/2 inhibition with sotagliflozin across the full spectrum of ejection fraction, including heart failure with preserved ejection fraction. In: Presented at the American College of Cardiology 2021 Scientific Sessions, May 17, 2021.

28. Arnott C, Huang Y, Neuen BL, et al. The effect of canagliflozin on amputation risk in the CANVAS program and the CREDENCE trial. Diabetes Obes Metab. 2020;22:1753-66.

29. Koepsell $\mathrm{H}$. The $\mathrm{Na}^{+}$-D-glucose cotransporters SGLT1 and SGLT2 are targets for the treatment of diabetes and cancer. Pharmacol Ther. 2017;170:148-65. 